\title{
Human Capital Distribution, Growth and Convergence
}

\author{
Danilo Guaitoli * \\ Universitat Pompeu Fabra ${ }^{\dagger}$
}

August 2000

*This is a revised version of part of my $\mathrm{PhD}$ dissertation (Guaitoli (1994)). I want to thank José Scheinkman, Gary Becker and Michael Woodford for precious comments, suggestions and encouragement; thanks also to Alberto Bisin, Afonso Mello Franco and audiences at the University of Chicago, Universitá Bocconi, CEPREMAP, Ecole des Ponts, Service d'Etude de l'Activite Economique, Universidad Carlos III de Madrid, ITAM, IGIER, Universitat Pompeu Fabra, University of Southern California, Simposio de Analisis Economico (Barcelona, 1993) and the conference on Income Distribution and Economic Development (Madrid, 1994). Financial support from Ente Einaudi, Bradley Foundation and the Human Capital and Mobility program of the EEC is gratefully acknowledged.

${ }^{\dagger}$ Dept. of Economics, Universitat Pompeu Fabra, Trias Fargas 25-27, 08005 Barcelona, Spain. E-mail: guaitoli@upf.es 


\begin{abstract}
This paper studies the dynamic relationship between distribution and endogenous growth in an overlapping generations model with accumulation of human and physical capital. It is shown how human capital can determine a relationship between per capita growth rates and inequality in the distribution of income. Family background effects and spillovers in the transmission of human capital generate a dynamics in which aggregate variables depend not only on the stock, but also on the distribution of human capital. The evolution of this distribution over time is then characterized under different assumptions on private returns and the form of the externality in the technology for human capital. Conditions for existence, uniqueness and stability of a constant growth equilibrium with a stationary distribution are derived. Increasing returns, idiosyncratic abilities and the possibility of poverty traps are explicitely characterized in a closed form solution of the equilibrium dynamics, showing the role played by technology and preferences parameters.
\end{abstract}

Keywords: income distribution, human capital, growth.

JEL: O40, D31. 


\section{Introduction}

The aim of this paper is to characterize the dynamic relationship between distribution and growth in an overlapping generations model with human capital accumulation as the engine of growth. In particular the focus is on the role of family background and aggregate spillovers, how through these channels inequality in the distribution of human capital affects per capita growth rates and levels, and how this distribution evolves over time.

Although the nature of the exercise is theoretical, its motivations are both theoretical and empirical. The use of representative agent endogenous growth models is supposedly a fair approximation if distributional effects have no relevant effect on aggregate variables. It is shown in this paper that this is true only in particular cases. In order to account for the diversity of growth experiences in economies with similar preferences and technology, our analysis suggests that inequality in the distribution of human capital is relevant to explain how economies with similar initial per capita income may end up on very different equilibrium paths.

There has been recently increasing empirical evidence suggesting a negative relationship between growth rates and inequality. Persson and Tabellini (1991) find negative coefficients for some measure of inequality in growth regressions for a subset of countries. World Bank data also show (with few exceptions) a neat contrast between Asia's miracle economies (like South Korea, Singapore, Taiwan, Hong Kong, Japan), which have experienced in the past 30 years high growth associated with low and declining inequality, as compared to African and Latin American countries (like Zambia, Ghana, Sudan, Peru, Argentina) where high inequality has been associated with very poor growth performance. Our model is an attempt to explain these stylized facts pointing to human capital as the crucial variable in the link between distribution and growth. It also provides an explicit analytic characterization of this dynamic relationship.

For a class of functional forms it is possible to characterize the dynamics of a model with human and physical capital, deriving conditions for existence, uniqueness and stability of a constant growth equilibrium with no inequality. A link between inequality and stochastic dominance is used to define a relationship between growth rates and inequality, and to check how robust the results are to the form of the externality. The negative sign of the relationship between inequality and growth depends on two forms of concavity in the technology for human capital: concavity in parental levels of human capital and concavity of the aggregator function which defines the external effects. The first type of concavity is also required for convergence to a stationary distri- 
bution. Only when individual returns are constant, inequality is irrelevant for aggregate growth rates and levels.

Closed form solutions for the equilibrium dynamics are obtained, checking the role of different preferences and technology specifications and incorporating additional features like idiosyncratic abilities and increasing returns. The conditions (in terms of the initial distribution) for persistent growth and the possibility of poverty traps, both at the aggregate and at the individual level, are characterized.

The content of this paper is related to the early work by Becker and Tomes (1979, 1986), which introduced human capital, family background and individual abilities at the center of the theory. A fairly large literature has developed more recently on the links between distribution and growth, mainly focusing on financial markets imperfections and political economy models. Some papers are formally closer to our approach, more directly related to human capital issues: Tamura (1991) on income convergence; Glomm and Ravikumar (1992) on private and public education; Benabou (1996) on stratification and community structure; Galor and Tsiddon (1997) on technological externalities and Kuznets curve-type dynamics.

The empirical relevance of family background and parental education on human capital accumulation and children's earnings has been documented by several papers, e.g.: Behrman and Wolfe (1984) on Nicaragua; Heckman and Hotz (1986) on Panama, estimating that a 1-year increase in mother's education increases the son's earnings by 3-5 percent; Lam and Schoeni (1993) on Brazil, where after controling for the worker's own schooling, having a university-educated vs. an illiterate father means a 20 percent wage advantage; Neal and Johnson (1996) on US black-white wage gaps, primarily reflecting a skill gap which in turn can be traced (in a relevant part) to differences in family background and parental education; Peraita and Sanchez (1997) on Spain, who also find family background affecting schooling attainment.

The rest of the paper is organized as follows. Section 2 describes the basic model and derives some general results on steady state properties and convergence. Sections 3 characterizes the relationship between growth rates and inequality along the transition path, deriving closed form solutions for the dynamics of the distribution and per capita variables, including idiosyncratic ability shocks and increasing returns, analyzing the possibility of poverty traps and non-monotonic equilibrium paths. Section 4 extends the analysis checking the sensitivity of the results to the specification of externalities, the degree of altruism, the form and timing of the investment, the utility function. All the proofs are contained in the Appendix. 


\section{Distribution, growth and convergence}

Consider an overlapping generation model where each generation is composed by a continuum $[0,1]$ of individuals. Agents differ only for the endowment of human capital $h_{t}$ which they receive as children. In the first period of adult life they allocate their unit of time between work and children's education. The income earned ( $w_{t} h_{t}$ per hour of work) is allocated between consumption at $t$ and savings for consumption at time $t+1$ (when old). Savings are invested in physical capital $\left(k_{t+1}\right)$ generating a return $r_{t+1}$ (assume complete depreciation).

Children's human capital $h_{t+1}$ depends on a productivity parameter $\theta_{t}$ (which may differ across individuals), on the fraction of time $a_{t}$ invested by parents, on the parental level of human capital $h_{t}$ (the family background effect), and on the average level of human capital $H_{t}$ (which measures the aggregate spillovers), according to the technology:

$$
h_{t+1}=\theta_{t} a_{t}^{\nu} h_{t}^{\delta} H_{t}^{\gamma}
$$

where $\nu, \delta$ and $\gamma$ are non-negative.

In this section we begin to study as a benchmark the case of constant returns to scale in the inputs that can be accumulated $\left(h_{t}\right.$ and $\left.H_{t}\right)$, with a common productivity parameter (so the only source of inequality is given by different family backgrounds). Therefore: $\theta_{t}=\theta$ for every agent; $\nu, \delta, \gamma \in(0,1)$ and $\gamma=1-\delta$.

Altruism that motivate parents to invest in their children's education may depend either on the utility of the children or on the children's level of human capital. The main difference for the investment decision is that in the first case parents take fully into account the effect of children's human capital both on their income and on the family background of successive generations: i.e. although the parental level of human capital is predetermined, the family background spillover is internalized. The effects on $H_{t+1}$ however will not be

internalized: a single individual will take aggregate spillovers that depend on the state of society as a whole as given.

The recursive problem in the dynastic case (superscripts denoting birth dates when necessary) is:

$$
v\left(h_{t}, H_{t}\right)=\max \left\{\ln c_{t}^{t-1}+\alpha \ln c_{t+1}^{t-1}+\beta v\left(h_{t+1}, H_{t+1}\right)\right\}
$$




$$
\begin{aligned}
\text { s.t. } \quad c_{t}^{t-1} & =\left(1-s_{t}\right)\left(1-a_{t}\right) w_{t} h_{t} \\
c_{t+1}^{t-1} & =r_{t+1} k_{t+1} \\
k_{t+1} & =s_{t}\left(1-a_{t}\right) w_{t} h_{t} \\
h_{t+1} & =\theta a_{t}^{\nu} h_{t}^{\delta} H_{t}^{1-\delta} \\
a_{t}, s_{t} & \in[0,1] .
\end{aligned}
$$

From the first order and envelope conditions a constant solution for $a_{t}$ and $s_{t}$ is obtained:

$$
\begin{aligned}
a_{t} & =\frac{\beta \nu}{1+\beta \nu} \equiv a \\
s_{t} & =\frac{\alpha}{1+\alpha} \equiv s .
\end{aligned}
$$

The evolution of the individual levels of capital is thus given by

$$
\begin{aligned}
h_{t+1} & =\theta a^{\nu} h_{t}^{\delta} H_{t}^{1-\delta} \\
k_{t+1} & =s(1-a) w_{t} h_{t} .
\end{aligned}
$$

Human and physical capital are used to produce goods with the technology

$$
Y_{t}=(1-a)^{\eta} H_{t}^{\eta} K_{t}^{1-\eta}
$$

where $H_{t} \equiv \int h_{t} d G_{t}\left(h_{t}\right)$ and $K_{t} \equiv \int k_{t} d F_{t}\left(k_{t}\right)$.

Profit maximization gives competitive prices

$$
\begin{aligned}
& w_{t}=(1-a)^{\eta} \eta\left(\frac{H_{t}}{K_{t}}\right)^{-(1-\eta)} \\
& r_{t}=(1-a)^{\eta}(1-\eta)\left(\frac{H_{t}}{K_{t}}\right)^{\eta}
\end{aligned}
$$


Substituting into (3)

$$
\begin{aligned}
h_{t+1} & =\theta a^{\nu} H_{t}^{1-\delta} h_{t}^{\delta} \\
k_{t+1} & =s(1-a)^{\eta+1} \eta\left(\frac{H_{t}}{K_{t}}\right)^{-(1-\eta)} h_{t} .
\end{aligned}
$$

Normalize the first equation dividing it by the average $H_{t+1}$ and define $z_{t} \equiv$ $h_{t} / H_{t}$ to get

$$
z_{t+1}=\frac{\theta a^{\nu}}{\left(H_{t+1} / H_{t}\right)} z_{t}^{\delta} .
$$

Remark 1 The policy function (7) at each $t$ is the same for every agent, it is strictly increasing and concave, starting from the origin, and the slope satisfies the Inada conditions.

By averaging (6) we obtain growth rates for human and physical capital

$$
\begin{aligned}
\frac{H_{t+1}}{H_{t}} & =\theta a^{\nu} E\left(z_{t}^{\delta}\right) \\
\frac{K_{t+1}}{K_{t}} & =s(1-a)^{\eta+1} \eta\left(\frac{H_{t}}{K_{t}}\right)^{\eta}
\end{aligned}
$$

which combine to determine the rate of growth of per capita income:

$$
\begin{aligned}
1+g_{t} & =\frac{Y_{t+1}}{Y_{t}}=\left(\frac{H_{t+1}}{H_{t}}\right)^{\eta}\left(\frac{K_{t+1}}{K_{t}}\right)^{1-\eta} \\
& =C\left(\frac{H_{t}}{K_{t}}\right)^{\eta(1-\eta)}\left[E\left(z_{t}^{\delta}\right)\right]^{\eta}
\end{aligned}
$$

where $C \equiv\left(\theta a^{\nu}\right)^{\eta}\left(s(1-a)^{\eta+1} \eta\right)^{1-\eta}$ and $E\left(z_{t}^{\delta}\right) \equiv \int h_{t}^{\delta} d G_{t}\left(h_{t}\right)$. 
Proposition 1 In the economy described, there exists a unique stationary equilibrium with complete equality, constant growth rate $g$ for $Y, H$ and $K$, and a constant $(H / K)$; the steady state growth rate is given by

$$
g=\max \left\{\theta a^{\nu}-1,0\right\}
$$

with $g>0$ (persistent growth) iff $\theta>1 / a^{-\nu}$.

The stationary growth rate $g$ depends positively on the productivity of human capital technology $\theta$, on the returns to investment $\nu$, and on the degree of altruism $\beta$ (cf. (2)).

Proposition 2 For any initial distribution with bounded support the economy converges to the stationary equilibrium of Prop. 1.

\section{Transitional dynamics, growth rates and in- equality}

In this section the joint dynamics of per capita levels and distribution is derived (showing in particular the dependence of per capita growth rates and levels on distribution); the conditions for persistent growth and the possibility of poverty traps, both at the aggregate and individual level, are characterized in terms of the initial distribution; the properties of the equilibrium paths, in particular the non-monotonicity of per capita variables, are discussed.

\subsection{Stochastic dominance and inequality}

Consider again equation (9) for per capita growth rates:

$$
1+g_{t}=C\left(\frac{H_{t}}{K_{t}}\right)^{\eta(1-\eta)}\left[E\left(z_{t}^{\delta}\right)\right]^{\eta} .
$$

For given per capita levels, is there any relation between growth rates and inequality in the distribution of human capital (the distribution of physical capital at time $t$ is determined by the distribution of $\left.h_{t-1}\right)$ ? Were the distribution concentrated on two levels (rich and poor), the concavity of $z^{\delta}$ would clearly show a decrease in $E\left(z_{t}^{\delta}\right)$ (and hence a lower growth rate) when the 
distance between rich and poor increase (for given mean). We can generalize such conclusion to any distribution if we define an increase in inequality as a mean-preserving spread of the distribution in the sense of second order stochastic dominance.

Definition 1 Given two cumulative distributions $G_{1}(z), G_{2}(z)$ with bounded support, for which $E_{1}(z)=E_{2}(z)=1$, we say that $G_{2}$ displays greater inequality (in the sense of second order stochastic dominance) if

$$
\int_{\underline{z}}^{y}\left[G_{1}(z)-G_{2}(z)\right] d z \leq 0 \quad \forall y \in[\underline{z}, \bar{z}],
$$

where $\underline{z}=\min \left\{\underline{z}_{1}, \underline{z}_{2}\right\}, \bar{z}=\max \left\{\bar{z}_{1}, \bar{z}_{2}\right\}$ (i.e. more density on the tails, cf. Rothschild and Stiglitz 1970).

Proposition 3 At any time $t$, for given per capita levels, greater inequality in the distribution of human capital (as defined above) implies a lower per capita growth rate.

\subsection{Increasing returns and ability shocks: the lognor- mal model}

We want to extend the analysis to study the consequences of introducing additional sources of inequality (individual abilities or luck) and increasing returns to scale. We also want to characterize more explicitly the transition path of the economy when these features are included. As in Glomm and Ravikumar (1992), we will focus on the class of lognormal distributions, for which we can derive an explicit recursive, reduced form for the dynamic system allowing for idiosyncratic shocks and increasing returns.

Consider a lognormal (cumulative) distribution $G_{t}\left(h_{t}\right)$, with $\left(\mu_{t}, \sigma_{t}^{2}\right)$ denoting mean and variance of $\ln h_{t}$. Here $\mu_{t}$ picks the median level of $h_{t}$, while

$\sigma_{t}^{2}$ measures inequality (Gini coefficients and Lorenz rankings for lognormal distributions depend only on $\sigma$ ). Then in equilibrium

$$
\begin{aligned}
H_{t} & =\int h_{t} d G_{t}\left(h_{t}\right) \\
& =\exp \left\{\mu_{t}+(1 / 2) \sigma_{t}^{2}\right\}
\end{aligned}
$$


We also assume an iid lognormal distribution for $\theta_{t}$ :

$$
\ln \theta_{t} \sim N\left(\mu_{\theta}, \sigma_{\theta}^{2}\right)
$$

so $\Theta=\exp \left\{\mu_{\theta}+(1 / 2) \sigma_{\theta}^{2}\right\}$ is the mean of $\theta_{t}$. As for physical capital, only the stock $K_{t}$ matters, not the distribution. Under these assumptions, since the dynamics is given by a loglinear system, $h_{t+1}$ will also have a lognormal distribution.

The dynamics of aggregate variables is then given by a two-dimensional, non-autonomous first-order linear system of the form

$$
X_{t+1}=M X_{t}+C_{t}
$$

where $X_{t}$ is a column vector with elements $\ln H_{t}$ and $\ln K_{t}$, and $C_{t}$ depends on the inequality in the distribution of human capital.

At this point one could perform numerical exercises, calibrating the model for some parameter values and generating the sequences that solve the system for different initial conditions. Alternatively, we can simplify the model by reducing it to a one-dimensional system, for which we can obtain explicit analytic solutions with a clearer idea of the role played by some crucial parameters. Abstracting from physical capital and considering a version with only human capital should preserve the qualitative features of the model, since it is clear from the previous section that the driving force of the dynamics is the distribution of human capital, while the distribution of physical capital does not play an autonomous role.

Under the assumptions made

$$
\begin{aligned}
\ln h_{t+1} & =\ln \theta_{t}+\nu \ln a+\gamma \ln H_{t}+\delta \ln h_{t} \\
\mu_{t+1} & =\ln A+\gamma \ln H_{t}+\delta \mu_{t} \\
& =\ln A+(\gamma+\delta) \mu_{t}+(\gamma / 2) \sigma_{t}^{2} \\
\sigma_{t+1}^{2} & =\delta^{2} \sigma_{t}^{2}+\sigma_{\theta}^{2}
\end{aligned}
$$

where $\ln A \equiv \mu_{\theta}+\nu \ln a$. 
Solving the last equation backward

$$
\sigma_{t}^{2}= \begin{cases}\delta^{2 t} \sigma_{0}^{2}+\frac{1-\delta^{2 t}}{1-\delta^{2}} \sigma_{\theta}^{2} & \text { if } \delta \neq 1 \\ \sigma_{0}^{2}+t \sigma_{\theta}^{2} & \text { if } \delta=1\end{cases}
$$

with

$$
\lim _{t \rightarrow \infty} \sigma_{t}^{2}= \begin{cases}\frac{1}{1-\delta^{2}} \sigma_{\theta}^{2} & \text { if } \delta<1 \\ \infty & \text { if } \delta \geq 1\end{cases}
$$

Combining and solving backward we can express the evolution of the average human capital in terms of the initial conditions:

$$
\begin{aligned}
\ln H_{t} & =\mu_{t}+(1 / 2) \sigma_{t}^{2} \\
& =(\gamma+\delta)^{t} \Phi_{t}
\end{aligned}
$$

where

$$
\begin{aligned}
\Phi_{t}= & \mu_{0}+\frac{1-(\gamma+\delta)^{-t}}{\gamma+\delta-1} \ln A+\frac{\sigma_{0}^{2}}{2\left(\gamma+\delta-\delta^{2}\right)}\left[\gamma+\left(\delta-\delta^{2}\right)\left(\frac{\delta^{2}}{\gamma+\delta}\right)^{t}\right]+ \\
& +\frac{\sigma_{\theta}^{2}}{2\left(1-\delta^{2}\right)}\left[\frac{\gamma\left(1-\delta^{2}\right)}{(\gamma+\delta-1)\left(\gamma+\delta-\delta^{2}\right)}-\frac{1-\delta}{\gamma+\delta-1}(\gamma+\delta)^{-t}+\right. \\
& \left.-\frac{\delta-\delta^{2}}{\left.\gamma+\delta-\delta^{2}\right)}\left(\frac{\delta^{2}}{\gamma+\delta}\right)^{t}\right]
\end{aligned}
$$

if $\gamma+\delta>1$, and

$$
\begin{aligned}
\Phi_{t}=t & {\left[\ln A+\frac{(1-\delta) \sigma_{\theta}^{2}}{2\left(1-\delta^{2}\right)}\right]+\frac{\left(\delta-\delta^{2}\right)\left(1-\delta^{2 t}\right) \sigma_{\theta}^{2}}{2\left(1-\delta^{2}\right)^{2}}+\mu_{0}+} \\
& +\left[\frac{(1-\delta)\left(1-\delta^{2 t}\right)}{1-\delta^{2}}+\delta^{2 t}\right] \frac{\sigma_{0}^{2}}{2}
\end{aligned}
$$


if $\gamma+\delta=1$.

Recursively, the law of motion of average human capital can be written as

$$
H_{t+1}=a^{\nu} \Theta \exp \left\{-\frac{\delta(1-\delta)}{2} \sigma_{t}^{2}\right\} H_{t}^{\gamma+\delta}
$$

with growth rate

$$
\begin{aligned}
g_{t} & =\ln H_{t+1}-\ln H_{t} \\
& =\nu \ln a+\ln \Theta+(\gamma+\delta-1) \ln H_{t}-\frac{\delta(1-\delta)}{2} \sigma_{t}^{2} .
\end{aligned}
$$

Remark 2 If family background has a decreasing marginal effect $(\delta<1)$ there is a stationary limit distribution, which is no longer degenerate on the mean but has a positive level of inequality as a function of the distribution of abilities or luck. Inequality decreases monotonically over time (as in the previous section) if the initial level is higher than the steady state level (14); but it can be increasing towards the steady state if it is initially lower, because idiosyncratic variance cumulates through the family background. If $\delta \geq 1$ inequality increases without bound.

Remark 3 The equilibrium path of per capita income (from (15)) depends on the initial distribution of human capital (i.e. on inequality $\sigma_{0}^{2}$ ), both in the short and in the long run, unless $\delta=1$. In particular, the same condition $(\delta<1)$ for the existence of a stationary distribution implies that growth rates at any time $t$ depend negatively on inequality (from (17)).

\subsection{Persistent growth vs. poverty trap}

We characterize now the conditions under which $\lim _{t \rightarrow \infty} H_{t}=+\infty$ (persistent growth) or $\lim _{t \rightarrow \infty} H_{t}=0$ (poverty trap).

In the case of constant returns to scale $(\gamma+\delta=1)$, the condition for persistent growth involves only the structural parameters (including the distribution

of abilities) and not the initial distribution of $h$. Taking the limit of (15), the condition for persistent growth is 


$$
\Theta>\left(1 / a^{\nu}\right) \exp \left\{\frac{\delta \sigma_{\theta}^{2}}{2(1+\delta)}\right\} .
$$

In the case of increasing returns to scale $(\gamma+\delta>1)$ we have the possibility of poverty traps dependent on the initial distribution. Income distribution (i.e. the distribution of human capital) can have permanent effects on growth rates, not only on levels. If $\delta<1$, persistent growth requires both a sufficiently high stock of human capital and a relatively low inequality in the distribution of that human capital:

Proposition $4^{1}$ With $\delta<1, \exists H^{\star}$ and $\hat{\sigma}_{0}^{2}=\sigma\left(H_{0}\right)$ s.t. : persistent growth iff $H_{0}>H^{\star}$ and $\sigma_{0}^{2}<\hat{\sigma}_{0}^{2}$.

Here, given $H_{t}$, growth rates are inversely related to $h_{t}$, i.e. families with less human capital on average grow faster. The concavity in $h_{t}$ implies that the average of individual growth rates is smaller, the greater the dispersion in the distribution of $h_{t}$. If inequality is too large, the economy will not grow fast enough to escape poverty.

The crucial factors in this relationship are again diminishing returns in parental human capital and the presence of externalities. If in fact the marginal effect of family background were non-decreasing, inequality would have either no effect on long run growth or even a positive effect. In particular, as the following proposition shows, $\delta=1$ is the only case in which distribution would not matter at all, because individual growth rates would depend only on the average $H_{t}$ and the ability shocks $\theta_{t}$. On the other hand, if increasing returns from aggregate spillovers were in part internalized at the individual level $(\delta>$ $1, \gamma>0$ ), growth rates would be positively related to individual levels; hence some inequality might help overcoming a poverty trap (unless $\delta$ or $H_{0}$ are very large, in which case persistent growth would occur independently of the initial distribution). If there were no aggregate spillovers $(\gamma=0)$, individual growth rates would depend only (positively) on parental levels and individual abilities or luck; since these are uncorrelated, there would always be a positive measure of families growing fast enough to sustain per capita income growth.

Proposition 5 Define $\bar{\delta} \equiv \frac{1+\sqrt{1+4 \gamma}}{2}$. Then (i) if $\delta=1, \exists \underline{H}$, s.t. for any $\sigma_{0}^{2}$ persistent growth iff $H_{0}>\underline{H}$.

\footnotetext{
${ }^{1}$ See figure 1, the stable manifold $S M$.
} 
(ii) if $1<\delta<\bar{\delta}$, when $H_{0}>H^{\star}$ persistent growth for any $\sigma_{0}^{2}$, when $H_{0}<H^{\star}$ persistent growth iff $\sigma_{0}^{2}>\hat{\sigma}_{0}^{2}$;

(iii) if $\delta>\bar{\delta}$, persistent growth for any $\sigma_{0}^{2}$ and any $H_{0}$;

(iv) if $\gamma=0$ and $\delta>1$, persistent growth for any $\sigma_{0}^{2}$ and any $H_{0}$.

Having derived conditions for persistent growth at the aggregate level, we prove now that these conditions are necessary and sufficient, with the aggregate externality, to make every family (even the poorest) escape poverty in the limit.

Proposition 6 If $\gamma>0$ (spillovers), for each individual family $\lim _{t \rightarrow \infty} h_{t}=+\infty$ iff $\lim _{t \rightarrow \infty} H_{t}=+\infty$; if $\gamma=0$ (no spillovers), each family has a positive probability of persistent growth (increasing in the level of $h_{t}$ ) as well as a positive probability of falling in a poverty trap.

With economy-wide spillovers the development process will eventually include the whole population. However the dynamics at the bottom of the distribution are quite different in the $\delta<1$ case (positive growth from the beginning, both in absolute and relative terms) relative to the $\delta>1$ case (families with low $h$ may be trapped in poverty for many generations and even when they get richer in absolute terms, they still lose ground in relative terms). Without aggregate spillovers growth becomes dualistic: even if there is individual mobility (as long as abilities and luck are not perfectly correlated with family background), a fraction of the population will eventually account for most of the wealth of the economy while others will be trapped in poverty.

\subsection{Transition}

Even when persistent growth is possible, inequality may slow down the aggregate path of the economy for many generations, i.e. the equilibrium path may be non-monotonic. In other words, there are initial conditions for which the economy will experience a phase of negative growth rates and decline, followed by a path of persistent growth. The converse is also true: for other initial conditions the economy will go through some periods of growth which later on turns into decline and permanent stagnation.

Proposition $7^{2}$ For any $\delta>0, \gamma \geq 0, \exists\left(H_{0}, \sigma_{0}^{2}\right)$ and a corresponding time $T$ s.t. $g_{t}<0$ for $t<T$ and $g_{t}>0$ for $t>T$. For any $\delta \in(0,1), \gamma>0$, $\exists\left(H_{0}, \sigma_{0}^{2}\right)$ and a corresponding time $T$ s.t. $g_{t}>0$ for $t<T$ and $g_{t}<0$ for $t>T$.

\footnotetext{
${ }^{2}$ See figure 1.
} 


\section{Extensions}

We want to extend the analysis of the previous sections and check the robustness of the results with respect to different features of the model (of preferences and technology), like the form of the externalities, the type of altruism, the form and timing of investment.

\subsection{Different forms of externality}

The results of the previous sections can be generalized further if we allow the externality in the technology for human capital to enter in a form different from the arithmetic mean considered so far. In particular, the result of a negative

relation between growth rates and inequality holds if the externality is defined by an aggregator of the form

$$
Z_{t}=F\left[\int f\left(h_{t}\right) d G_{t}\left(h_{t}\right)\right]
$$

with $F^{\prime}>0$ and $f$ concave.

In this case the equivalent expressions for (6), (8) and (9) are

$$
\begin{aligned}
h_{t+1} & =\theta a^{\nu} Z_{t}^{1-\delta} h_{t}^{\delta} \\
\frac{H_{t+1}}{H_{t}} & =\theta a^{\nu} E\left(z_{t}^{\delta}\right)\left(\frac{Z_{t}}{H_{t}}\right)^{1-\delta} \\
1+g_{t} & =C\left(\frac{H_{t}}{K_{t}}\right)^{\eta(1-\eta)}\left[E\left(z_{t}^{\delta}\right)\right]^{\eta}\left(\frac{Z_{t}}{H_{t}}\right)^{\eta(1-\delta)}
\end{aligned}
$$

(the equations for $k_{t}$ and $K_{t+1} / K_{t}$ don't change).

If we increase inequality in the sense of second order stochastic dominance, $Z_{t}$ will decrease as well as $E\left(z_{t}^{\delta}\right)$, lowering $g_{t}$. For example, if $Z_{t}$ takes the form of a CES (as in Benabou (1992))

$$
Z_{t}=\left[\int h_{t}^{\frac{\sigma-1}{\sigma}} d G_{t}\left(h_{t}\right)\right]^{\frac{\sigma}{1-\sigma}}
$$


our result will hold for any $\sigma>0$ (i.e. as long as different levels of $h$ are complementary; if $\sigma<0$ the levels of $h$ are substitutes and inequality increases $Z_{t}$, although the final effect on $g_{t}$ is ambiguous because $E\left(z_{t}^{\delta}\right)$ still declines).

An intuitive interpretation of these results is the following: a concave aggregator may refer to a situation where people at the low end of the distribution slow down substantially the progress of more advanced agents; a convex function, on the contrary, may refer to a situation where the more advanced part of the distribution (the frontier of knowledge) is the crucial one in determining the productivity of the whole system. In a more articulated multi-sector model it could be the case that different sectors fall into a different category of spillover effects; the lack of a clear cut implication for the aggregate relation between growth and distribution would still be consistent with substantial effects (possibly in opposite directions) at a more disaggregated level.

Finally, we could have additional spillovers also in the production of goods, but this would not change qualitatively the results: growth of per capita output is determined by the dynamics of the distribution of human capital, so the relevant spillovers are the ones in human capital accumulation.

\subsection{Altruism and the form of investment}

We want to show that qualitatively the dynamics is robust to some changes in the form of preferences, the form and timing of investment, and the addition of spillovers in the production of goods.

The technology for human capital implies that as long as $a_{t}$ is constant in equilibrium, the law of motion for $h_{t}$ is linear in logarithms. The characterization of the evolution of the distribution and per capita variables and the analytic results for the class of lognormal distributions will not change qualitatively if the value of $a_{t}$ is just a different constant. With log preferences this feature is preserved even if we make different assumptions about the degree of altruism, the parameters of the technology and the form or timing of the investment decisions.

For example, a constant $a_{t}$ is chosen by agents whether investment decisions are made before or after observing the realization of $\theta_{t}$. As for altruism, in alternative to the dynastic utility

$$
v(h, H)=\max \left\{u(a, c)+\beta v\left(h^{\prime}, H^{\prime}\right)\right\}
$$

we could have a more limited altruism when parents only care about the level of human capital bequeted to their children: 


$$
U\left(a, c, h^{\prime}\right)=u(a, c)+\beta \ln h^{\prime} .
$$

The solution is again a constant $a_{t}$ given by

$$
a=\frac{\beta \nu}{1+\alpha+\beta \nu} .
$$

If, instead of parents, young individuals themselves invest their time (as alternative to leisure) in the accumulation of human capital, producing and consuming goods only in the second part of their life, the solution (abstracting from physical capital) is a constant $a_{t}$ given by

$$
\begin{array}{rlr}
a & =\frac{\alpha \nu(1+\beta \delta)}{1+\alpha \nu(1+\beta \delta)} \quad \text { with altruism } \\
& =\frac{\alpha \nu}{1+\alpha \nu} \quad \text { with no altruism. }
\end{array}
$$

If on the other hand we change the log linear structure of the model, the fraction of time invested in human capital will no longer be constant (unless the production functions of goods and human capital are the same), but will depend on the levels. For example, if preferences take the form

$$
U\left(c_{t}, h_{t+1}\right)=\frac{1}{1-\sigma}\left(c_{t}^{1-\sigma}+\beta h_{t+1}^{1-\sigma}\right), \quad \sigma>0
$$

with constraints

$$
\begin{aligned}
c_{t} & =\left(1-a_{t}\right) h_{t} \\
h_{t+1} & =\theta_{t} a_{t}^{\eta} h_{t}^{\delta} H_{t}^{1-\delta}
\end{aligned}
$$

the solution for $a_{t}$ is

$$
a_{t}=a\left(h_{t} / H_{t} ; \theta_{t}\right)
$$

where for $\sigma<1, \quad \partial a / \partial(h / H)<0$ and $\partial a / \partial \theta>0$; for $\sigma>1$, $\partial a / \partial(h / H)>0$ and $\partial a / \partial \theta<0$. 
Alternatively, consider the case of agents with log preferences producing and consuming goods (instead of leisure) in both periods. Assume that agents can smooth their consumption over the life cycle (but not across generations) by borrowing and lending at the exogenous rate $r$. Also assume that human capital is productive only in the second period of life (after some investment has been made). Then $a_{t}$ and the consumption profile are obtained by solving: ${ }^{3}$

$$
\begin{aligned}
\max \left\{\ln c_{t}+\right. & \left.\alpha \ln c_{t+1}\right\} \\
\text { s.t. } \quad c_{t}+\frac{1}{1+r} c_{t+1} & =y_{t}+\frac{1}{1+r} y_{t+1} \\
y_{t} & =1-a_{t} \\
y_{t+1} & =h_{t+1} \\
h_{t+1} & =\theta\left(a_{t} h_{t}\right)^{\delta} H_{t}^{1-\delta} .
\end{aligned}
$$

The solution is given by

$$
\begin{gathered}
a_{t}=\min \left\{\hat{a}_{t}, 1\right\} \\
\hat{a}_{t}=\left(\frac{\delta \theta}{1+r}\right)^{\frac{1}{1-\delta}} H_{t} h_{t}^{\frac{\delta}{1-\delta}} .
\end{gathered}
$$

Even with $\delta<1$, individual growth rates may be increasing in $h_{t}$ at low levels (when $a_{t}$ is increasing), and decreasing for higher $h_{t}$ (when $a_{t}$ is in a corner at 1$)$. For $\delta \in\left(\frac{1}{2}, 1\right)$, individual growth rates are in fact increasing in the lower range and decreasing in the upper range of $h_{t}$. The dynamics of inequality need not be monotonic, but may be increasing at low levels of income and then decreasing at higher levels (the so called Kuznets curve). Furthermore, if increasing returns in $h_{t}$ prevail at low levels, some inequality might be needed in poor economies to escape poverty. ${ }^{4}$ Although the empirical relevance of these patterns is still debated, these results are consistent with a technology in which family background and aggregate spillovers play a role.

\footnotetext{
${ }^{3}$ Altruism is not necessary here since the agents investing also benefit directly from the return of the investment.

${ }^{4}$ Cf. Galor and Tsiddon (1997) as an example of a different model with similar results.
} 


\section{Conclusions}

In this paper I have provided an explicit characterization of the dynamic interrelation between distribution of human capital and growth. Such links are obtained in the absence of any redistributive policy or distortionary taxation. Family background effects and spillovers in the accumulation of human capital are sufficient to generate a dependence of growth rates on the distribution of human capital. Inequality tends to have negative effects on growth when there is concavity in parental levels of human capital and in the aggregation that defines the spillovers. On the other hand, some inequality can increase growth rates if increasing returns dominate both at the individual level and in the determination of the externality. In terms of human capital it is reasonable to believe that sooner or later individuals will face decreasing returns. This is also required for convergence to a stationary distribution. If aggregate returns to scale are constant, eventually inherited inequalities will disappear and the stationary distribution will depend only on factors such as abilities and luck; even so, the process of convergence may be very slow (one period here is equivalent to half the life span of a generation). Growth rates will become independent of initial conditions but permanent differences in per capita levels will result from different initial distributions. If there are increasing returns at the aggregate level, income distribution may even have permanent effects on growth rates because of the possibility of poverty traps.

Supply-side effects dominate here (mainly through human capital technology), while the specification of preferences, the form and timing of the investment do not qualitatively change the results. Uninsured risk of the type considered only makes the limit distribution non degenerate, suggesting that while incentive constraints on financial contracts may be quite relevant for growth, they are not the only channel through which distribution and growth are related.

Finally, further extensions and applications of this analysis can be related to the study of efficient redistribution policies and their impact on growth and distribution; the interactions between saving behavior and human capital investment in a context where (non-negative) bequests of other assets are possible; the study of particular forms of spillovers as they relate to the structure of cities and local communities, the mobility of human capital, and the possibility of internalizing such spillovers. 


\section{References}

Becker, G., and N. Tomes, (1979). "An Equilibrium Theory of the Distribution of Income and Intergenerational Mobility," Journal of Political Economy, 87(6), 1153-1189.

Becker, G., and N. Tomes, (1986). "Human Capital and the Rise and Fall of Families," Journal of Labor Economics, 4, S1-S39.

Benabou, R., (1996). "Heterogeneity, Stratification and Growth: Macroeconomic Implications of Community Structure and School Finance," American Economic Review, 86(3), 584-609.

Behrman, J., and B. Wolfe, (1984). "The Socioeconomic Impact of Schooling in a Developing Country," Review of Economics and Statistics, 66, 296-303.

Galor, O., and D. Tsiddon, (1997). "The Distribution of Human Capital and Economic Growth," Journal of Economic Growth, 2, 93-124.

Glomm, G., and B. Ravikumar, (1992). "Public versus Private Investment in Human Capital: Endogenous Growth and Income Inequality," Journal of Political Economy, 100(4), 818-834.

Guaitoli, D., (1994). "Income Distribution, Financial Markets and Growth," unpublished Ph.D. dissertation, University of Chicago.

Heckman, J., and J. Hotz, (1986). "An Investigation of the Labor Market Earnings of Panamanian Males: Evaluating the Sources of Inequality," Journal of Human Resources, 21, 507-542.

Lam, D., and R. Schoeni, (1993). "Effects of Family Background on Earnings and Returns to Schooling: Evidence from Brazil," Journal of Political Economy, 101(4), 710-740.

Neal, D., and W. Johnson, (1996). "The Role of Premarket Factors in BlackWhite Wage Differences," Journal of Political Economy, 104(5), 869-895.

Peraita, C., and M. Sanchez, (1997). "The Effect of Family Background on Children's Level of Schooling Attainment in Spain," Universitat de Valencia, 
DT 97-06.

Persson, T., and G. Tabellini, (1991). "Is Inequality Harmful for Growth? Theory and Evidence," American Economic Review, 84(3), 600-621.

Rothschild, M., and J. Stiglitz, (1970). "Increasing Risk I: A Definition," Journal of Economic Theory, 2, 225-243.

Tamura, R., (1991). "Income Convergence in an Endogenous Growth Model," Journal of Political Economy, 99(3), 522-540. 


\section{Appendix}

Proof of Proposition 1. First note from (6) that the individual choice of $k_{t+1}$ is proportional to $h_{t}$, so convergence in human capital implies convergence in physical capital as well. It is also easy to see that in a constant growth equilibrium (in which $Y, H$ and $K$ all grow at the same constant rate $g$ ) the only stationary value of $z$ is $z=1$ (i.e. no inequality in human capital): in such case in fact, by the properties described in Remark 1, the function (7) has one stationary point (besides zero) and it is the same for all agents; since by definition the average of the $z$ 's is always 1 , it must be $z=1$.

In a steady state (with $z=1$ ) the two equations in (8) become

$$
\begin{aligned}
& 1+g=\theta a^{\nu} \\
& 1+g=s(1-a)^{\eta+1} \eta(H / K)^{\eta}
\end{aligned}
$$

with a unique interior solution

$$
\begin{aligned}
g & =\theta a^{\nu}-1 \\
(H / K) & =\left(\theta a^{\nu} / s(1-a)^{\eta+1} \eta\right)^{1 / \eta} .
\end{aligned}
$$

If $\theta a^{\nu}<1$ we have a corner solution at zero.

Proof of Proposition 2. Step 1: $z_{t} \rightarrow 1$ for every agent. Define $\underline{z}_{t}$ and $\bar{z}_{t}$ as the inf and sup (resp.) of the support of the $z_{t}$ distribution. Since by definition $E\left(z_{t}\right)=1 \quad \forall t, \quad \underline{z}_{t}<1<\bar{z}_{t} \forall t$. Also monotonicity and concavity of (7) imply $\underline{z}_{t+1}>\underline{z}_{t}$ and $\bar{z}_{t+1}<\bar{z}_{t} \quad \forall t \quad$ (otherwise all $z$ 's would move in the same direction, up or down, causing $E\left(z_{t+1}\right) \neq 1$ ). Furthermore, the ratio of any two $z$ 's (e.g. $\underline{z}_{t}$ and $\left.\bar{z}_{t}\right)$ evolves according to $\left(\underline{z}_{t+1} / \bar{z}_{t+1}\right)=\left(\underline{z}_{t} / \bar{z}_{t}\right)^{\delta}$, which converges monotonically to 1 for any $\left\{H_{t}\right\},\left\{K_{t}\right\}$. Hence all $z$ 's converge to 1 (convergence to the mean).

Step 2: aggregate variables converge to constant growth. Combining the two equations in (8) we have

$$
\frac{H_{t+1}}{K_{t+1}}=\frac{\theta a^{\nu}}{s(1-a)^{\eta+1} \eta} E\left(z_{t}^{\delta}\right)\left(\frac{H_{t}}{K_{t}}\right)^{1-\eta} .
$$

Given $E\left(z_{t}^{\delta}\right)$, the right hand side is strictly increasing and concave in $\left(H_{t} / K_{t}\right)$, starting from 0 with the slope satisfying the Inada conditions. Define $q^{\star} \equiv$ $(H / K)^{\star}$ as the stationary point when $z_{t}=1$. For any $H_{0} / K_{0} \in\left(0, q^{\star}\right), \quad \exists T$ 
finite s.t. $H_{t} / K_{t}>q^{\star}-\Delta \quad \forall t>T$ : in fact step 1 implies that $\exists T^{\prime}$ s.t. $E\left(z_{t}^{\delta}\right) \in[1-\epsilon, 1] \quad \forall t>T^{\prime}$, where $T^{\prime}$ and $\epsilon$ are taken to have the curve above intersecting the $45^{\circ}$ line to the right of $q^{\star}-\Delta$; after $T^{\prime}$, in a finite number of periods $H_{t} / K_{t}>q^{\star}-\Delta$ (and the same will hold thereafter). For $H_{0} / K_{0}>q^{\star}$ repeat the argument using the highest possible curve (with $E\left(z_{t}^{\delta}\right)=1$ ). Since it works for any $\Delta$ arbitrarly small, $H_{t} / K_{t} \rightarrow q^{\star}$. When $(H / K)$ and the distribution of $z$ 's converge, $Y_{t}, H_{t}$ and $K_{t}$ will grow at the same constant rate.

Proof of Proposition 3. From (9) $g_{t}$ is increasing in $E\left(z_{t}^{\delta}\right)$ which, for $\delta<1$, has the same properties of an expected utility. Therefore we can apply the result of Rothschild and Stiglitz (1970) according to which a mean-preserving spread in the distribution of a random payoff in the sense of second order stochastic dominance lowers the expected utility of risk averse agents. Here increasing inequality in that same sense lowers $E\left(z_{t}^{\delta}\right)$ and hence $g_{t}$.

Proof of Proposition 4. From (15), substituting $\mu_{0}$ from (10),

$$
\begin{aligned}
\lim _{t \rightarrow \infty} \Phi_{t} & =\Phi \\
& \equiv \ln H_{0}+\frac{\ln A}{\gamma+\delta-1}-\frac{\delta-\delta^{2}}{2\left(\gamma+\delta-\delta^{2}\right)} \sigma_{0}^{2}+\frac{\gamma \sigma_{\theta}^{2}}{2(\gamma+\delta-1)\left(\gamma+\delta-\delta^{2}\right)}
\end{aligned}
$$

since $\gamma+\delta>1$, persistent growth iff $\Phi>0$, which gives the upper bound on $\sigma_{0}^{2}$ as a function of $H_{0}$ (the line $S M$ in figure 1 ):

$$
\hat{\sigma}_{0}^{2}=\frac{2\left(\gamma+\delta-\delta^{2}\right)}{\delta-\delta^{2}}\left[\ln H_{0}+\frac{\ln A}{\gamma+\delta-1}+\frac{\gamma \sigma_{\theta}^{2}}{2(\gamma+\delta-1)\left(\gamma+\delta-\delta^{2}\right)}\right]
$$

when $H_{0}<H^{\star} \equiv A^{-\frac{1}{\gamma+\delta-1}} \exp \left\{-\frac{\gamma \sigma_{\theta}^{2}}{2(\gamma+\delta-1)\left(\gamma+\delta-\delta^{2}\right)}\right\}$ the upper bound is negative, so it cannot be satisfied by any $\sigma_{0}^{2}>0$.

Proof of Proposition 5. (i) From (13) and (15), $\lim _{t \rightarrow \infty} \Phi_{t}>0$ iff

$$
\ln H_{0} \equiv \mu_{0}+\frac{\sigma_{0}^{2}}{2}>-\frac{\ln A}{\gamma}-\frac{\gamma M \sigma_{\theta}^{2}}{2(\gamma+1)}
$$

where $M \equiv \sum_{j=0}^{\infty} j(\gamma+1)^{-j}$, which defines the threshold $\underline{H} \equiv A^{-\frac{1}{\gamma}} \exp \left\{-\frac{\gamma M \sigma_{\theta}^{2}}{2(\gamma+1)}\right\}$. 
(ii) Same as Proposition 4 but reverse inequality; (iii)-(iv) from (15), $\lim _{t \rightarrow \infty} \Phi_{t}=$ $+\infty$ for any $\sigma_{0}^{2}, H_{0}$.

Proof of Proposition 6. Solving the first equation in (12) backward $(\gamma>0)$

$$
\ln h_{t}=\delta^{t} \ln h_{0}+\frac{1-\delta^{t}}{1-\delta} \eta \ln a+\frac{1}{\delta} \sum_{j=0}^{t-1} \delta^{t-j} \ln \theta_{j}+\frac{\gamma}{\delta} \sum_{j=0}^{t-1} \delta^{t-j} \ln H_{j}
$$

using (15)

$$
\lim _{t \rightarrow \infty} \delta^{t} \sum_{j=0}^{t-1} \delta^{-j} \ln H_{j}=\lim _{t \rightarrow \infty} \delta^{t} \sum_{j=0}^{t-1}\left(\frac{\gamma+\delta}{\delta}\right)^{j} \Phi_{t}=+\infty
$$

iff $\lim _{t \rightarrow \infty} \Phi_{t}>0$ (condition for persistent per capita growth); if $\delta<1$ the term $\sum_{j=0}^{t-1} \delta^{t-j} \ln \theta_{j}$ is finite with probability one; if $\delta>1$ the term is also finite after collecting $\delta^{t}$. If $\gamma=0$ and $\delta>1$

$$
\ln h_{t}=\delta^{t}\left[\ln h_{0}+\frac{\left(1-\delta^{-t}\right) \eta \ln a}{\delta-1}+\frac{1}{\delta} \sum_{j=0}^{t-1} \delta^{-j} \ln \theta_{j}\right] ;
$$

the limit of the term in square brackets can be positive or negative according to the initial level $h_{0}$ and the realized history of shocks $\left\{\theta_{t}\right\}$.

Proof of Proposition 7. With constant returns to scale $(\gamma+\delta=1)$, since the growth rate depends negatively on inequality, it's clearly possible to start with high inequality and negative growth rates until, with declining inequality, the slope of the curve becomes greater than one (or viceversa, if the steady state is stagnation). With increasing returns to scale, the stationary points for $H_{t}$ (i.e. where $H_{t+1}=H_{t}$ ) are given by

$$
\ln H_{t}=\max \left[\ln H^{\star}, \frac{-\ln \left(a^{\nu} \Theta\right)}{\gamma+\delta-1}+\frac{\delta(1-\delta)}{2} \sigma_{t}^{2}\right]
$$

where $H^{\star}$ is the stationary point at $\sigma_{t}^{2}=0$. If $\delta<1$, the stationary level of inequality is given by (14). Both curves are drawn in figure 1; the stable manifold (from Prop. 4) is flatter than the stationary line above and it crosses it in one point: hence there exist regions from which non-monotonic paths arise. For $\delta>1$ the same analysis gives a similar result but only for paths with positive growth in the limit. 


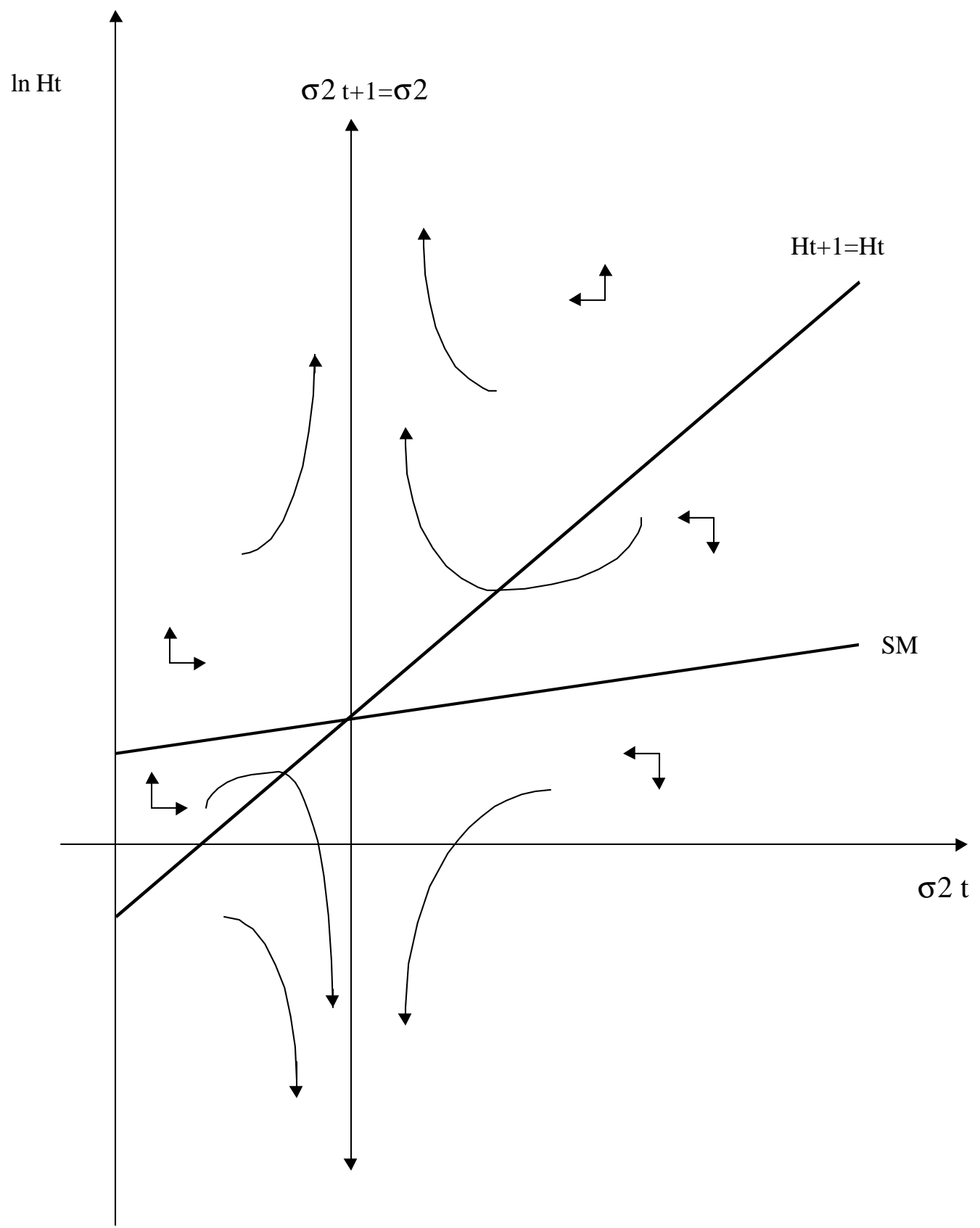

FIGURE 1 